\title{
Region-Based Clustering for Lung Segmentation in Low-Dose CT Images
}

\author{
Fernando C. Monteiro \\ Polytechnic Institute of Bragança, Portugal
}

\begin{abstract}
Lung segmentation in thoracic computed tomography scans is essential for the development of computer-aided diagnostic methods for identifying the lung diseases. Low-dose CT scans are increasingly utilized in lung studies, but segmenting them with traditional threshold segmentation algorithms often yields less than satisfying results. In this paper we present a hybrid framework to lung segmentation which joints region-based information based on watershed transform with clustering techniques. The proposed method eliminates the task of finding an optimal threshold and the over-segmentation produced by watershed. We have applied our approach on several pulmonary low-dose CT images and the results reveal the robustness and accuracy of this method.
\end{abstract}

Keywords: Lung segmentation, Graph clustering, Watershed transform, Pulmonary CT image. PACS: $87.57 . \mathrm{nm}$

\section{INTRODUCTION}

Due to the fast and high technology progress in high resolution X-ray computed tomography (CT) scanners, multislice helical CT of the thorax is widely used to evaluate numerous lung diseases. To provide useful and reliable information, most such CAD methods need to identify the lung boundaries within the images, a preprocessing step known as lung segmentation, before computer analysis can take place. Given the large size of CT datasets, manually segmenting the lungs is tedious and prone to interobserver variations. This encourages many investigators to develop computer-aided diagnostic (CAD) methods to assist radiologists [1,2].

Several research groups have proposed and developed techniques to segment the lung regions of pulmonary CT images. Sluimer's et al. survey paper [3] summarizes the methods of analysing thoracic scans using computer, including segmentation of various pulmonary structures, registration of chest scans and applications of CAD.

Pixel-based methods taking into account the large gradient value between the lungs and surrounding tissues are the common way in lung segmentation $[1,4]$. However, CT lung density depends on many factors such as image acquisition protocol, X-ray dosage, subject tissue volume, volume air, and physical material properties of the lung. In particular, for low-dose lung CT images, there are more linear artefacts by the beam hardening effect. These factors make lung segmentation based on threshold technique difficult. So developing new accurate algorithms with no human interaction to precisely segment the lung is important.

Watershed algorithm is a popular approach calculating the boundaries of the catchment basins which are situated at the high gradient point forming a gradient image. Homogeneous regions are clustered based on the edge detection and arithmetical morphology. Often, the watershed transform experiences over-segmentation problem resulting in the segmentation of unwanted regions. To overcome this issue, marker based watershed transform is used rather than conventional watershed algorithm [5]. The markers are applied to the gradient image to avoid over-segmentation where it decreases the regional minima connecting them with the region of interest.

The proposed methodology in this paper has four major stages. First, we create an over-segmented image (atomic regions) based on the watershed transform of the gradient image. In the second stage, the over-segmented image will be the input for the region similarity graph (RSG) construction. Next, we apply a graph clustering approach on the RSG. This framework integrates edges and region-based segmentation with spectral-based clustering through the watershed transform. Finally, the morphological opening operator is used both to eliminate small objects inside and outside the lung and to enhance the separation between distinct regions so as to make the lung border recognition easier.

The combination of watershed and clustering methods solves the weaknesses of each method. Rather than clustering single feature points we will cluster small regions, confident that the underlying primitive regions are reliable. Our approach actually prefers the objects to be over-segmented into a number of smaller regions to ensure that a minimal amount of background is connected to any of the lung regions. 
The remainder of this paper is organized as follows. Next section gives a description of the methods used in this paper. Followed by the experimental results and discussion section. The concluding remarks are given in the last section.

\section{LUNG SEGMENTATION}

Image segmentation plays a crucial role in lung modelling. The goal of image segmentation research is to increase the reliability, accuracy, precision, and to reduce the computational cost of the algorithms.

Direct application of the watershed segmentation algorithm generally leads to over-segmentation due to noise and other local irregularities of the gradient. A practical solution to this problem is to limit the number of allowable regions by incorporating a preprocessing stage designed to bring additional knowledge into the segmentation procedure.

A critical issue in watershed techniques is known to be over-segmentation i.e. the tendency to produce too many basins. Several methods have been proposed in the literature to reduce the spurious boundaries created due to noise and produce a meaningful segmentation.

In this work we provide three methods to overcome this problem. First, bilateral anisotropic filtering can be applied to remove noise from the image. Secondly, some of the weakest edges are removed by a gradient minima suppression process known as pre-flooding. This concept uses a measure of depth of a certain basin. Prior to the transform, each catchment basin is flooded up to a certain height above its bottom, i.e. the lowest gradient magnitude and it can be thought as a flooding of the topographic image at a certain level (flooding level). The third one, handles to control over-segmentation by merging the atomic regions with a graph-based clustering approach.

Spectral-based methods use the eigenvectors and eigenvalues of a matrix derived from the pairwise similarities of features (pixels or regions). This effect is achieved by constructing a fully connected graph. Considering all pairwise pixel relations in an image may be too computational expensive. Unlike other well known clustering methods [6] which use down-sampling pixel-based to construct the graph, our method is based on selecting links from a weighted undirected graph $G=(V, E, W)$ based on a region similarity graph where each node corresponds to an atomic region.

The proposed region similarity graph structure takes advantage on region-based representation. The set of nodes $V$ are represented by the centroid of each atomic region. The sets of links $E$ and link weights $W$ represent, respectively, relationships and similarity measures between pair of regions. Each region $r_{i}$ represents a small group of pixels where the centroid $\bar{x}_{i}$ is utilized as a node of the graph.

In almost all the graph-based approaches proposed in the literature the spatial distance cue is also used to compute the similarity between graph nodes. However, during our experiments, we noted that such cue is responsible for the partition of homogeneous areas in the image - an issue commonly associated to normalized cut algorithm. Instead, we use intervening contours [9] which are equivalent to spatial distance without suffering from the same problems. For each pair of nodes, node similarity is inversely correlated with the maximum contour energy encountered along the line connecting the centroids of the regions. If there are strong contours along a line connecting two centroids, these atomic regions probably belong to different segments and should be labelled as dissimilar.

Let $i$ and $j$ be two atomic regions and the orientation energy $O E^{*}$ between them, then the intervening contours contribution to the link weight is given by:

$$
w_{i c}(i, j)=\exp \left[-\frac{\max _{t \in \operatorname{line}(i, j)}\left\|O E^{*}\left(\bar{x}_{i}, \bar{x}_{j}\right)\right\|^{2}}{\sigma_{i c}^{2}}\right],
$$

where line $(i, j)$ is the line between centroids $\bar{x}_{i}$ and $\bar{x}_{j}$ formed by $t$ pixels.

The mean intensity of each node contributes for the link weight according to the following function:

$$
w_{I}(i, j)=\exp \left(-\frac{\left(I_{\bar{x}_{i}}-I_{\bar{x}_{j}}\right)^{2}}{\sigma_{I}^{2}}\right) .
$$

These cues are combined in a final link weight similarity function, with the values $\sigma_{i c}$ and $\sigma_{I}$ selected in order to maximize the dynamic range of $\mathbf{W}$ :

$$
\mathbf{W}(i, j)=w_{i c}(i, j) \cdot w_{I}(i, j)
$$

To compute the similarity matrix the current approach uses only image brightness and magnitude gradient. Additional features such as texture, could be added to the similarity criterion. This may slow the construction of the RSG but the rest of the algorithm will proceed with no change. 


\section{EXPERIMENTAL RESULTS}

The low-dose pulmonary CT images used in this paper are obtained from the the Early Lung Cancer Action Program (ELCAP) Public Lung Image Database which were made by collaboration between the ELCAP and VIA research groups of Cornell University [8]. This database contains CT lung images of 50 subjects. The number of image slices for different subjects varies. The average number of image slices per subject is around 260 . We selected 176 low-dose CT images from case W0011-20000101, as the test set. The conditions of these low-dose CT images are as follows: the tube voltage is $120 \mathrm{KV}$, the tube current is $80 \mathrm{~mA}$, and the slice thickness is $1.25 \mathrm{~mm}$. The images obtained in this condition are stored with size of $512 \times 512$ pixels. Each pixel is 16 bits in size and 0.6-0.75 mm resolution. The images are in greyscale mode, stored in DICOM format.

Each CT scan consists of a variable number of slices not all of which contain useful information for our purposes. Actually, the first and last slices where the lungs appear do not usually coincide with the first and last slices of the scan; also, the order numbers of these slices vary from one examination to another. Therefore, we can assume that a few initial as well as a few final slices might not contain any lung images. We call these slices useless slices. On the other hand, within the remaining slices, called useful slices (in our case, from slice 12 to slice 187), we can still identify three different groups corresponding, respectively, to the upper, middle and lower parts of the lung volume. Figure 1 shows three slices selected from different parts of the chest to show the accuracy of this technique.

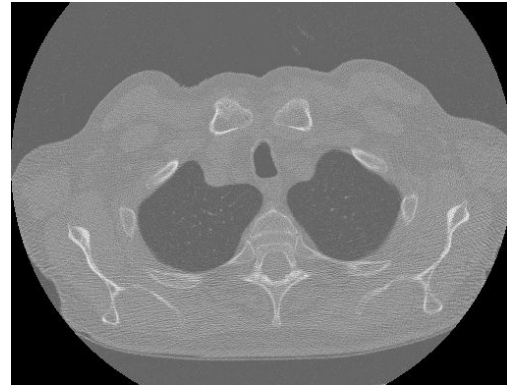

(a)

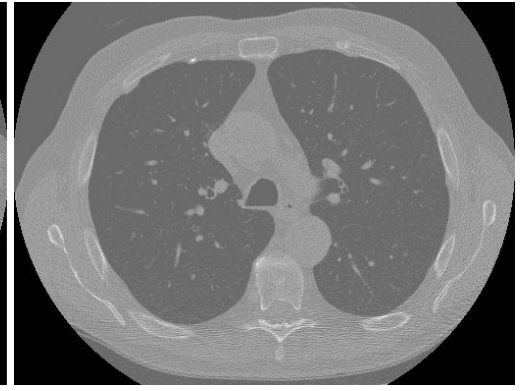

(b)

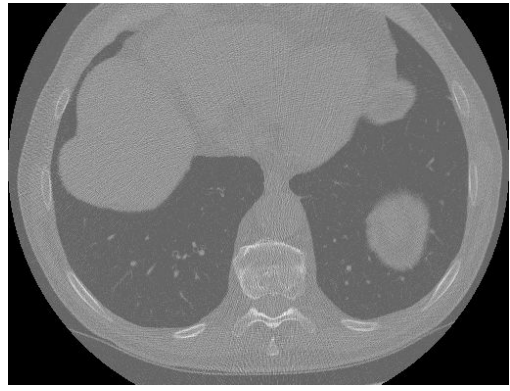

(c)

FIGURE 1. Original CT lung image of subject w0011 from ELCAP [8], slice: (a) 24, (b) 76, (c) 158.

Segmentation result of slice 76 directly using watershed algorithm is shown in Fig. 2.(a) and from it we can find that serious over-segmentation makes the result meaningless, even with some pre-processing as in Fig. 2.(b).

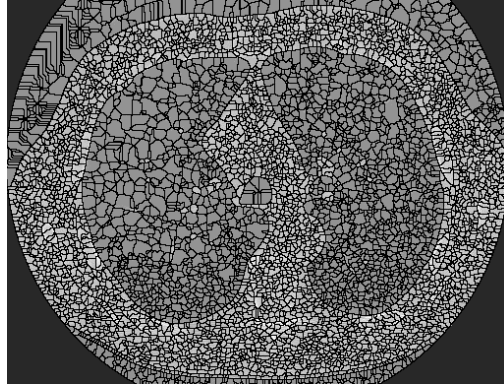

(a)

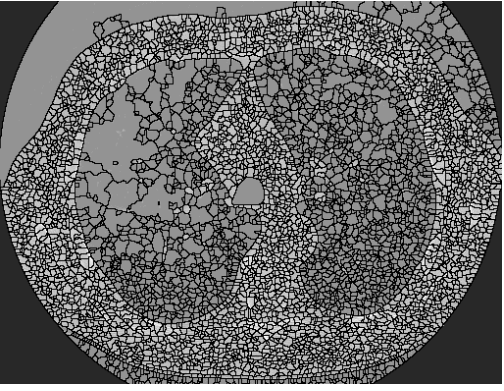

(b)

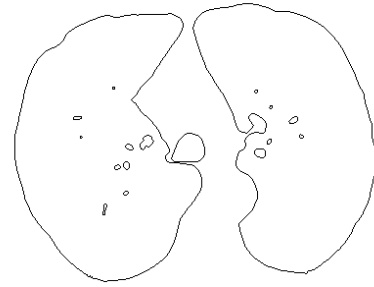

(c)

FIGURE 2. (a) Atomic regions from watershed. (b) Atomic regions from watershed with pre-processing. (c) Image segmentation borders before morphological operators.

The lung segmentation results for the slices in Fig. 1 are shown in Fig. 3. Comparing the segmented lung regions with the original image confirms that our approach separates accurately the pulmonary regions.

The region to examine for nodule detection has now been highly reduced. Indeed, we have eliminated all the external structures. At this point, we can, e.g., perform the 3D reconstruction of the lungs (see Fig. 4) using a program for volumetric reconstruction. 


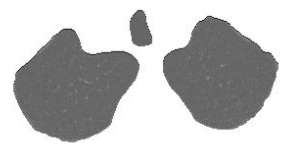

(a)

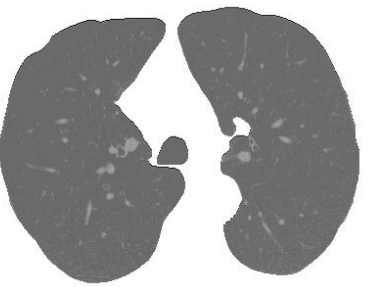

(b)

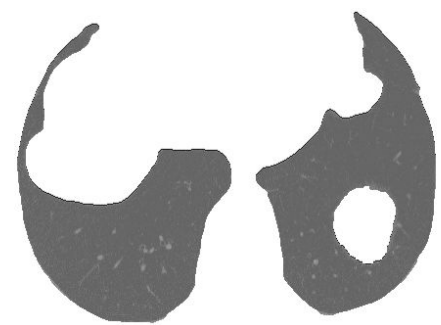

(c)

FIGURE 3. Lung tissue extracted images corresponding to the slices in Fig. 1, respectively.

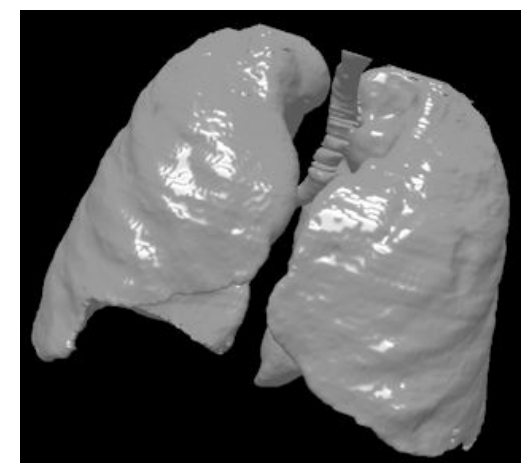

FIGURE 4. 3D reconstruction of the lungs.

\section{CONCLUSION}

The recent studies demonstrate that the lung segmentation method as a preprocessing step for CAD systems may affect CAD results. We have described a method for automated segmentation and reconstruction of pulmonary parenchyma. Aiming at the limitation of watershed segmentation, this paper presents an algorithm of cooperation between watershed transformation and graph clustering operation. We applied this technique on a subset of 2D CT lung images from the ELCAP database corresponding to different parts of the chest and the results are visually acceptable. This method can be also utilized to detect pulmonary nodules in the segmented lung region by removing the the final stage of the proposed method. At the moment, our method is at an experimental stage and needs to be evaluated against ground truth images produced by radiologists.

\section{REFERENCES}

1. S. G. Armato, and W. F. Sensakovic, Automated lung segmentation for thoracic CT: impact on computer-aided diagnosis, Academic Radiology 11(9), pp. 1011-1021, (2004).

2. R. Wiemker, P. Rogalla, T. Blaffert, et al., Aspects of computer-aided detection (CAD) and volumetry of pulmonary nodules using multislice CT, The British Journal of Radiology 78, pp. S46-S56, (2005).

3. I. C. Sluimer, A. M. R. Schilham, M. Prokop, and B. van Ginneken, Computer analysis of computed tomography scans of the lung: a survey, IEEE Transactions on Medical Imaging 25(4), pp. 385-405, (2006).

4. S. Hu, E.A. Hoffman, and J.M. Reinhardt, Automatic lung segmentation for accurate quantitation of volumetric X-ray CT images, IEEE Transactions on Medical Imaging 20(6), pp. 490-498, (2001).

5. R. Shojaii, J. Alirezaie, and P. Babyn, Automatic lung segmentation using watershed transform, in International Conference of Image Processing, Genova, Italy, vol. II, pp. 1270-1273 (2005).

6. J. Shi and J. Malik, Normalized cuts and image segmentation, IEEE Transactions on Pattern Analysis and Machine Intelligence 22(8), pp. 888-905, (2000).

7. E.M. van Rikxoort, B. de Hoop, S. van de Vorst, M. Prokop, B. van Ginneken, Automatic segmentation of pulmonary segments from volumetric chest CT scans, IEEE Transactions on Medical Imaging 28(4), pp. 621-630, (2009).

8. ELCAP Public Lung Image Database, VIA and ELCAP Labs, Cornell University. http://www.via.cornell.edu/lungdb.html.

9. T. Leung and J. Malik, Contour continuity in region-based image segmentation. In H. Burkhardt and B. Neumann, editors, Procedings European Conference in Computer Vision, volume 1, pp. 544-549, Freiburg, Germany, June 1998. Springer-Verlag. 